\title{
Ubiquitin-like modifier-activating enzyme 7 as a marker for the diagnosis and prognosis of breast cancer
}

\author{
MENG LIN ${ }^{1}$, YANQING $\mathrm{LI}^{2}$, SHANSHAN QIN ${ }^{3}$, YAN JIAO ${ }^{4}$ and FANG HUA ${ }^{5}$ \\ ${ }^{1}$ Department of Breast Surgery, The First Hospital of Jilin University; ${ }^{2}$ Department of Pathophysiology, \\ College of Basic Medical Science, Jilin University, Changchun, Jilin 130021; ${ }^{3}$ Department of Radiology, Affiliated \\ Hospital of Qingdao University, Qingdao, Shandong 266000; ${ }^{4}$ Department of Hepatobiliary and Pancreatic Surgery; \\ ${ }^{5}$ Cardiovascular Center, The First Hospital of Jilin University, Changchun, Jilin 130021, P.R. China
}

Received July 9, 2019; Accepted January 14, 2020

DOI: $10.3892 / \mathrm{ol} .2020 .11406$

\begin{abstract}
Ubiquitin-like modifier-activating enzyme 7 (UBA7) is a specific E1-like ubiquitin-activating enzyme involved in interferon-stimulated gene 15 (ISG15) conjugation. UBA7 expression has been reported to be notably decreased in lung cancer. The present study aimed to investigate the changes in UBA7 expression in breast cancer and the association between UBA7 expression and clinical characteristics, and to elucidate the diagnostic and prognostic significance of UBA7 in breast cancer. The clinical data and RNA-sequencing expression values of 1,104 patients with breast cancer were downloaded from The Cancer Genome Atlas database. The associations between UBA7 expression and clinical characteristics were determined using $\chi^{2}$ and Fisher's exact tests. UBA7 expression values were divided into low and high groups using the optimal cut-off value, as determined by the overall survival (OS) value identified via a receiver operating characteristic (ROC) curve analysis, to further study the association between UBA7 expression and clinical characteristics. The diagnostic capability of UBA7 was assessed via ROC analysis, and Kaplan-Meier curve and Cox regression analyses were performed to determine the prognostic value of UBA7. The results demonstrated that UBA7 expression was decreased in breast cancer, and significant differences were observed between groups with regards to vital status, tumor classification, metastasis classification, histological type, sex, molecular subtype, and expression levels of progesterone receptor, estrogen receptor
\end{abstract}

Correspondence to: Dr Yan Jiao, Department of Hepatobiliary and Pancreatic Surgery, The First Hospital of Jilin University, 71 Xinmin Street, Changchun, Jilin 130021, P.R. China

E-mail: lagelangri1@126.com

Dr Fang Hua, Cardiovascular Center, The First Hospital of Jilin University, 71 Xinmin Street, Changchun, Jilin 130021, P.R. China E-mail: yaya7013@163.com

Key words: breast, carcinoma, diagnosis, prognosis, ubiquitin-like modifier-activating enzyme 7
(ER) and human epidermal growth factor receptor 2. Low and high UBA7 expression levels were associated with age, ER expression, menopause status, Tumor-Node-Metastasis classification stage, margin status, vital status, radiation therapy use, OS and relapse-free survival. Furthermore, patients with low UBA7 expression levels had a poor prognosis. UBA7 expression also demonstrated an ability to diagnose patients at all clinical stages. Taken together, the results indicated that UBA7 expression was significantly decreased in breast cancer, and was associated with clinical characteristics and prognosis. Thus, UBA7 can be deemed as a potential biomarker in breast cancer, and may serve as a target in treatment.

\section{Introduction}

Today, breast cancer is the second leading cause of cancer-associated mortality in women in the United States $(1,2)$. It is reported that 1 in 8 women (13\%) are expected to develop breast cancer in their lifetime in the United States $(1,2)$. Breast cancer is commonly divided into two types: Infiltrating lobular carcinoma, which originates in the cells of the lobules, and infiltrating ductal carcinoma, which originates in the ducts (2). A few subtypes originate in the stromal tissues, such as the fatty and fibrous connective tissues of the breast. There are four molecular subtypes of breast cancer, according to the expression levels of estrogen receptor (ER), progesterone receptor (PR), human epidermal growth factor receptor 2 (HER2) and Ki-67, including luminal A, luminal B, HER2-enriched and basal-like (2). The pathogenesis of breast cancer remains unclear; however, it is considered to begin with alterations at the genomic level (2). Different types of breast cancer have a wide range of tumor growth rates and variable clinical courses, thus potential biomarkers are required to aid with diagnosis and prognosis, and may also function as novel targets in the treatment of breast cancer.

Interferon-stimulated gene 15 (ISG15) is the first ubiquitin-like protein to be discovered and reported as a negative regulator in IFN- $\alpha / \beta$ immunity, and to play a key role in antivirus and antitumor defences $(3,4)$. ISG15 is upregulated by IFNs (5) and has the ability to conjugate with ubiquitin-associated proteins $(3,4)$. The conjugation process, known as 
interferon-stimulated gene 15 conjugation (ISGylation), includes three respective steps, namely activation, conjugation and ligation, and occurs in several cellular signaling pathways (6). A total of three classes of enzymes partake in this process, including an E1 activating enzyme [ubiquitin-like modifier-activating enzyme 7 (UBA7)], E2 conjugating enzymes, such as ubiquitin-carrier protein $\mathrm{H} 8$ (UBCH8) and ubiquitin-carrier protein $\mathrm{H6}$ (UBCH6), and E3 ligases, such as estrogen-responsive finger protein (EFP), and HECT domain and RCC1-like domain-containing protein 5 (HERC5) (7). Currently, UBA7 is the only known E1-activating enzyme (8), and ISGylation has the ability to both activate and inhibit the activity of target proteins (4).

UBA7, also referred to as ubiquitin-activating enzyme E1-like protein (UBE1L), is the specific E1-like ubiquitin-activating enzyme that functions in ISGylation (9). Currently, the only known biological function of UBA7 is catalysing ISGylation. Both type I IFN and retinoic acid can induce UBA7 expression (10). Most previous studies on the UBA7/ISG15 signaling pathway in cancer have focused on lung cancer (8-12). UBA7 is located on chromosome 3p21.3 and is considered to be a candidate tumor suppressor gene. The loss of allelic heterozygosity ( $\mathrm{LOH}$ ) on 3p21.3 has been observed in $70-80 \%$ of non-small cell lung cancer (NSCLC) and $90-100 \%$ of SCLC cases (8). Furthermore, UBA7 expression was demonstrated to be notably decreased in several lung cancer cell lines (11). A number of studies have indicated that UBA7 suppresses tumor growth by inhibiting cyclin D1 expression, and that it can downregulate epidermal growth factor receptor (EGFR) expression in human bronchial epithelial cells $(9,12)$. However, to the best of our knowledge, there are currently no studies on the change in UBA7 expression in breast cancer. Thus, the present study set out to determine whether UBA7 could function as a biomarker in breast cancer.

The present study evaluated UBA7 expression in breast cancer, while subtypes were also taken into consideration, and the potential association between UBA7 expression and clinical characteristics was analyzed. The efficiency of UBA7 expression in diagnosis was also assessed, along with its prognostic value using overall survival (OS) and relapse-free survival (RFS) as outcome measures.

\section{Materials and methods}

Data collection. The clinical data and RNA-sequencing (RNA-seq) expression values of patients with breast cancer were downloaded from The Cancer Genome Atlas (TCGA) database (https://cancergenome.nih.gov/), and the corresponding accession code was TCGA-BRCA. The RNA-seq by expectation-maximization expression values were used in the statistical analysis of RNA-seq data.

Statistical analysis. R software (version 3.5.2) and associated packages were used for data analysis $(13,14)$. Wilcoxon test, Kruskal Wallis test and Dunn's post-hoc test were used in this study. mRNA expression differences between groups of discrete variables were depicted as boxplots generated by the ggplot2 package (version 3.2.1) in R software, and the association between clinical characteristics and UBA7 expression was
Table I. Clinical patient characteristics.

Characteristics

Number of cases $(\%)$

Age
$<60$
$\geq 60$
Sex
N/A

Age, years

$<60$

$\geq 60$

$590(53.44)$

$514(46.56)$

N/A

$2(0.18)$

Female

$1090(98.73)$

Male

$12(1.09)$

Histological type

N/A

$3(0.27)$

Infiltrating ductal carcinoma

$790(71.56)$

Infiltrating lobular carcinoma

204 (18.48)

Other

107 (9.69)

Molecular subtype

N/A

255 (23.04)

Basal

142 (12.86)

HER2-enriched

67 (6.07)

Luminal A

422 (38.22)

194 (17.57)

Luminal B

24 (2.17)

Normal

$50(4.53)$

2 (0.18)

Indeterminate

239 (21.65)

Negative

813 (73.64)

PR status

N/A

51 (4.62)

Indeterminate

$4(0.36)$

Negative

345 (31.25)

Positive

704 (63.77)

HER2 status

N/A

183 (16.58)

Equivocal

$180(16.3)$

Indeterminate

$12(1.09)$

Negative

565 (51.18)

Positive

164 (14.86)

Menopause status

N/A

93 (8.42)

Indeterminate

34 (3.08)

40 (3.62)

Peri

706 (63.95)

Post

231 (20.92)

$\mathrm{T}$ classification

N/A

$2(0.18)$

$\mathrm{T} 1$

$281(25.45)$

$\mathrm{T} 2$

640 (57.97)

T3

138 (12.50)

T4

$40(3.62)$

$3(0.27)$

$\mathrm{N}$ classification

N/A

$2(0.18)$

NO 
Table I. Continued.

\begin{tabular}{l} 
Characteristics \\
\hline N1 \\
N2 \\
N3 \\
NX \\
M classification \\
N/A \\
M0 \\
M1 \\
MX \\
Stage \\
N/A \\
I \\
II \\
III \\
IV \\
X
\end{tabular}

Lymph node status

N/A
No
Yes

Margin status

N/A

Close

Negative

Positive

Vital status

N/A

Deceased

Living

Radiation therapy

N/A

No

Yes

$72(6.52)$

$31(2.81)$

$922(83.51)$

$79(7.16)$

$2(0.18)$

$155(14.04)$

$947(85.78)$

$102(9.24)$

$445(40.31)$

$557(50.45)$

Neoadjuvant treatment

N/A

No

1088 (98.55)

Yes

$13(1.18)$

Targeted molecular therapy

N/A

No

$525(47.55)$

$46(4.17)$

$533(48.28)$

Sample type

Metastatic

Primary tumor

$1097(99.37)$

Overall survival

N/A
No
Yes

$154(13.95)$

Relapse-free survival

N/A

$192(17.39)$

No

$816(73.91)$

$96(8.70)$
Table I. Continued.

Characteristics

Number of cases $(\%)$

$\begin{array}{ll}\text { UBA7 } & \\ \text { High } & 588(53.26) \\ \text { Low } & 516(46.74)\end{array}$

N/A, not available; ER, estrogen receptor; PR, progesterone receptor; HER2, human epidermal growth factor receptor 2; UBA7, ubiquitin-like modifier-activating enzyme 7.

assessed using $\chi^{2}$ and Fisher's exact tests. A receiver operating characteristic (ROC) curve generated by the pROC package (version 1.15.3) in $\mathrm{R}$ software was used to determine the diagnostic capability of UBA7. UBA7 expression levels were divided into low and high groups, according to the optimal cut-off value, as determined by OS analysis using the ROC curve. Kaplan-Meier curves were generated to compare OS and RFS between the low and high expression groups, and P-values were calculated using the log-rank test. Univariate Cox analysis was performed to select the variables associated with OS and RFS, and multivariate Cox analysis was performed to assess the effect of UBA7 expression on survival and other clinical characteristics. $\mathrm{P}<0.05$ was considered to indicate a statistically significant difference.

\section{Results}

Patient characteristics. Both the clinical data and RNA-seq expression data of 1,104 patients with breast cancer were downloaded from TCGA database. The detailed clinical characteristics, including molecular subtype, Tumor-Node-Metastasis stage (2), residual tumor status, survival status and therapy type, are presented in Table I.

Association between UBA7 expression and clinicopathological characteristics. Low UBA7 mRNA expression levels were observed in breast cancer tissues compared with those in normal tissues from the same patients $(\mathrm{P}=0.039$; Fig. 1A). Significant differences in UBA7 expression were also demonstrated according to: Vital status $\left(\mathrm{P}=2.1 \times 10^{-4}\right)$, $\mathrm{T}$ classification $(\mathrm{P}=0.025), \mathrm{M}$ classification $(\mathrm{P}=0.0003)$, histological type $\left(\mathrm{P}=1.6 \times 10^{-13}\right)$ and sex $(\mathrm{P}=0.016)$ (Fig. 1). Furthermore, UBA7 expression was indicated to be significantly associated with the expression of breast cancer-associated molecules, as determined by the molecular subtype of breast cancer $\left(\mathrm{P}=4.1 \times 10^{-11}\right.$; Fig. 2A), and the expression of $\mathrm{PR}\left(\mathrm{P}=8.3 \times 10^{-12}\right.$; Fig. $\left.2 \mathrm{~B}\right), \mathrm{ER}$ $\left(\mathrm{P}=1.2 \times 10^{-7}\right.$; Fig. 2C) and HER2 ( $\mathrm{P}=0.0089$; Fig. 2D). However, no significant associations were demonstrated between UBA7 expression and the use of radiation therapy ( $\mathrm{P}=0.43$; Fig. $2 \mathrm{E})$, neoadjuvant therapy $(\mathrm{P}=0.11$; Fig. $2 \mathrm{~F})$ or targeted molecular therapy ( $\mathrm{P}=0.92$; Fig. $2 \mathrm{G})$.

UBA7 expression was divided into high and low groups according to the cutoff point (9.542), and low UBA7 level was demonstrated to be associated with histological type $(\mathrm{P}=0.0005)$, molecular subtype $(\mathrm{P}=0.0005)$, and the expression of $\mathrm{ER}(\mathrm{P}=0.0005), \mathrm{PR}(\mathrm{P}=0.0005)$ and HER2 $(\mathrm{P}=0.0185)$, 

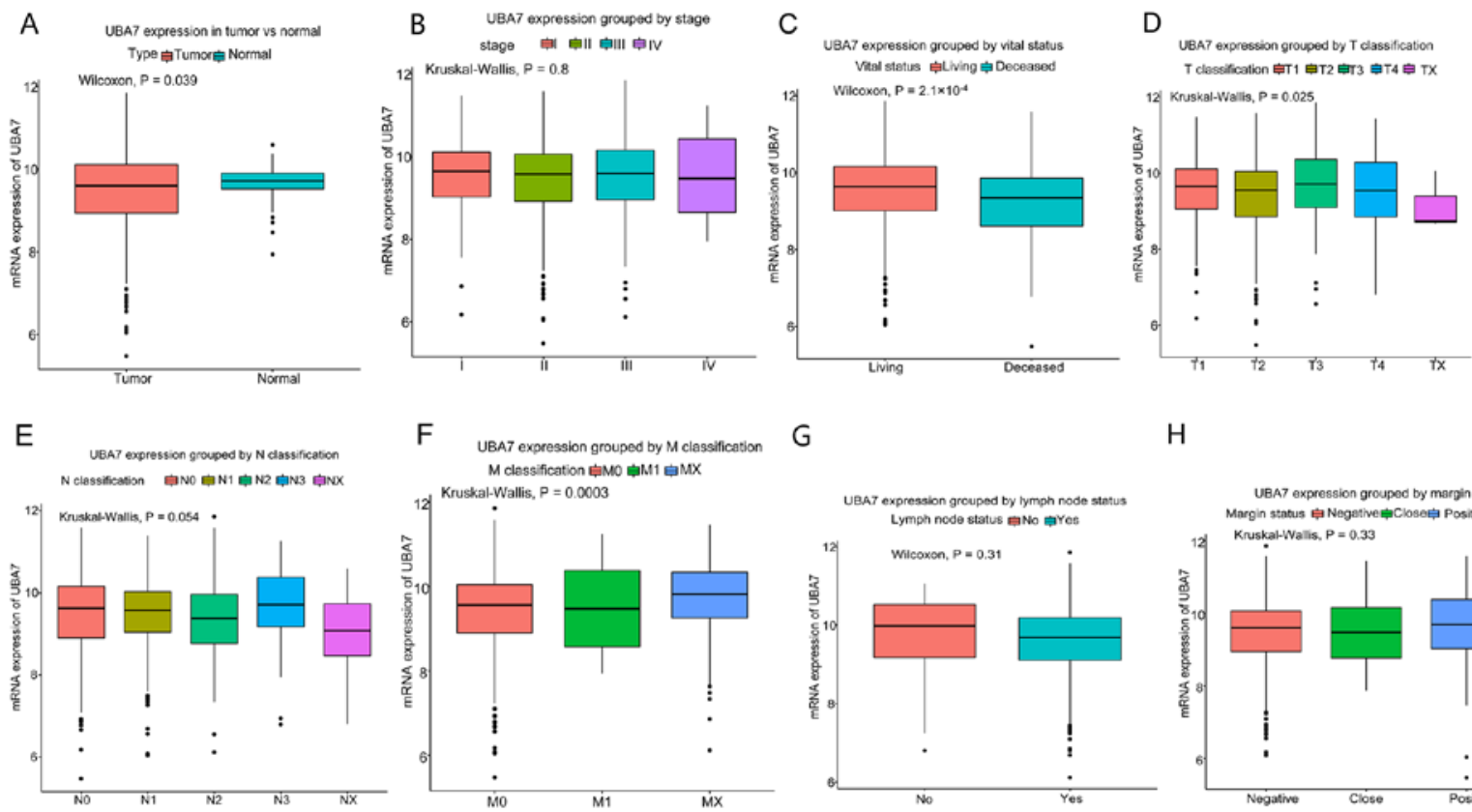

F UBA7 expression grouped by $M$ classinication G

$\mathrm{H}$
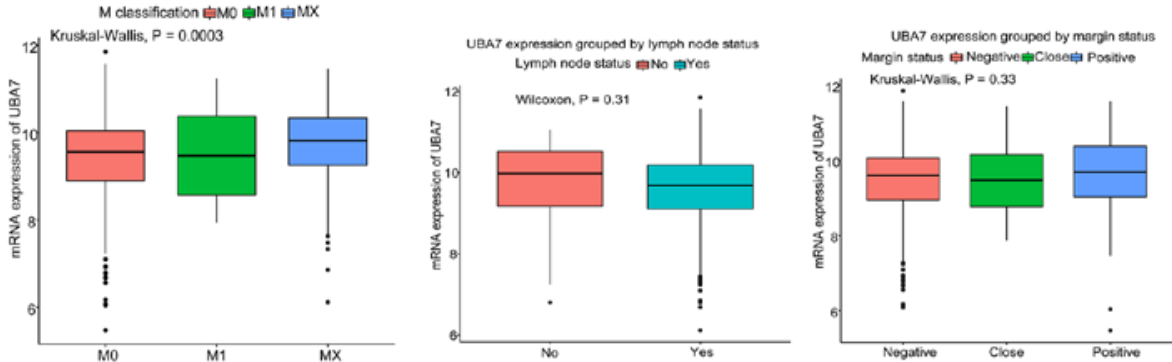

I

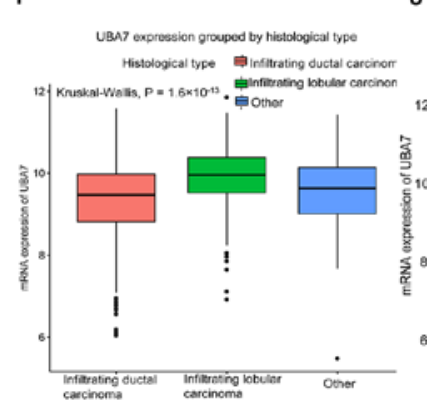

J

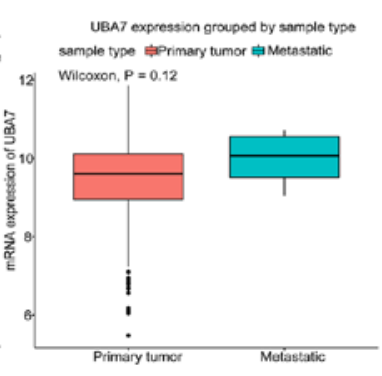

$\mathrm{K}$

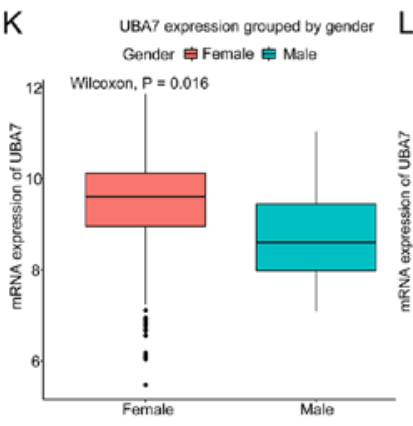

$\mathrm{L}$

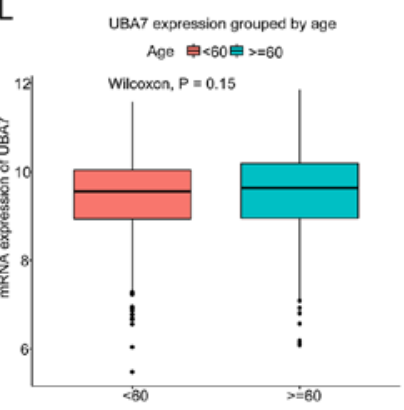

Figure 1. Differences in UBA7 expression according to clinicopathological characteristics. (A) UBA7 expression was lower in breast cancer tissues compared with that in normal tissues. Expression was significantly associated with (C) vital status, (D) tumor classification, (F) metastasis classification, (I) histological type and (K) gender. However, no significant association was observed with regard to (B) clinical stage, (E) N classification, (G) lymph node status, (H) margin status, (J) sample type and (L) age. UBA7, ubiquitin-like modifier-activating enzyme 7; T, tumor; M, metastasis.

A

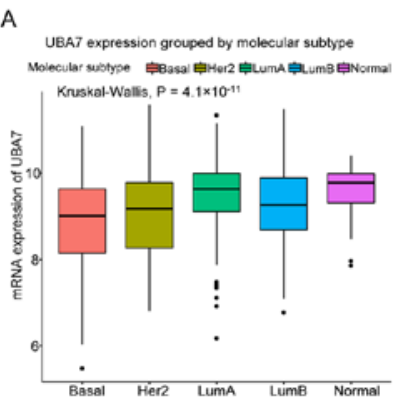

E UBA7 expression grouped by radiation theracy

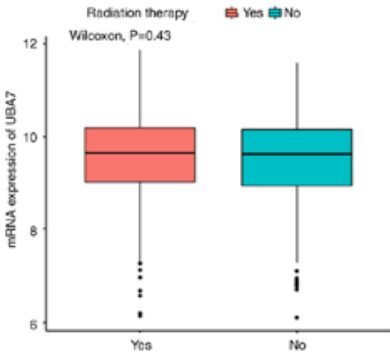

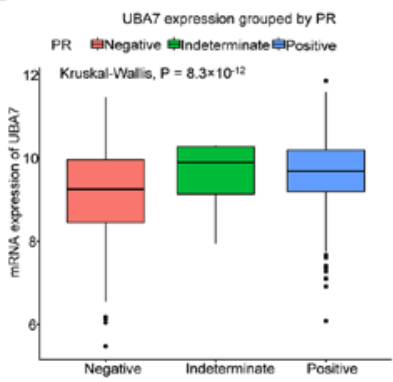

F UBA7 expression grouped by neosdjuant treatment

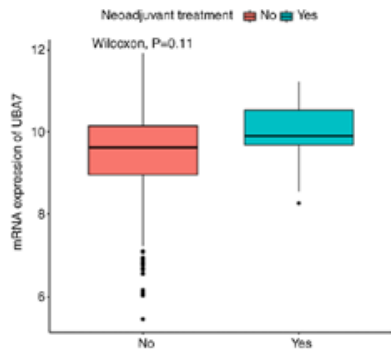

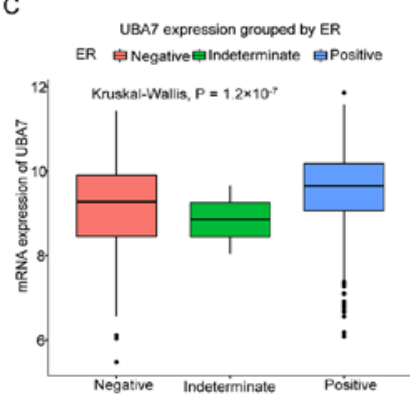

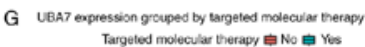

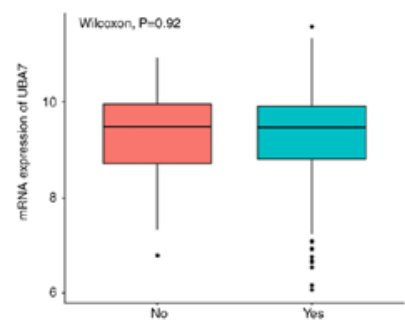

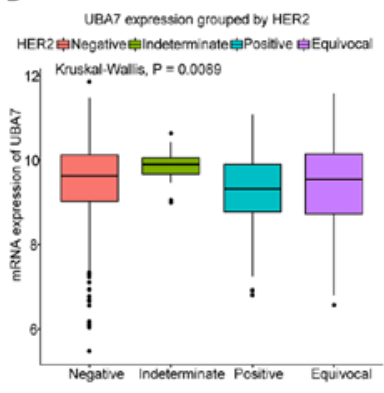

Figure 2. Differences in UBA7 expression according to clinicopathological characteristics. Significant differences were observed according to (A) molecular subtype of breast cancer, and (B) PR, (C) ER and (D) HER2 expression. However, no significant differences were observed according to (E) radiation therapy, (F) neoadjuvant treatment and (G) targeted molecular therapy. UBA7, ubiquitin-like modifier-activating enzyme 7; ER, estrogen receptor; PR, progesterone receptor; HER2, human epidermal growth factor receptor 2. 
Table II. Association between UBA7 mRNA expression and clinicopathological characteristics in patients with breast cancer.

\begin{tabular}{|c|c|c|c|c|c|}
\hline \multirow[b]{2}{*}{ Characteristics } & \multirow[b]{2}{*}{ Patients, n } & \multicolumn{2}{|c|}{ UBA7 mRNA expression } & \multirow[b]{2}{*}{$\chi^{2}$} & \multirow[b]{2}{*}{ P-value } \\
\hline & & High, n (\%) & Low, n (\%) & & \\
\hline Age, years & & & & 2.9564 & 0.0830 \\
\hline$<60$ & 589 & $299(51.02)$ & $290(56.2)$ & & \\
\hline$\geq 60$ & 513 & $287(48.98)$ & $226(43.8)$ & & \\
\hline Sex & & & & 3.8682 & 0.0785 \\
\hline Female & 1090 & $583(99.49)$ & $507(98.26)$ & & \\
\hline Male & 12 & $3(0.51)$ & $9(1.74)$ & & \\
\hline Histological type & & & & 43.5504 & $0.0005^{\mathrm{a}}$ \\
\hline Infiltrating ductal carcinoma & 790 & $377(64.33)$ & $413(80.19)$ & & \\
\hline Infiltrating lobular carcinoma & 204 & $150(25.6)$ & $54(10.49)$ & & \\
\hline Other & 107 & $59(10.07)$ & $48(9.32)$ & & \\
\hline Molecular subtype & & & & 37.2883 & $0.0005^{\mathrm{a}}$ \\
\hline Basal & 142 & $44(11.11)$ & $98(21.63)$ & & \\
\hline HER2-enriched & 67 & $26(6.57)$ & $41(9.05)$ & & \\
\hline Luminal A & 422 & $237(59.85)$ & $185(40.84)$ & & \\
\hline Luminal B & 194 & 75 (18.94) & $119(26.27)$ & & \\
\hline Normal & 24 & $14(3.54)$ & $10(2.21)$ & & \\
\hline ER status & & & & 17.7115 & $0.0005^{\mathrm{a}}$ \\
\hline Indeterminate & 2 & $1(0.18)$ & $1(0.2)$ & & \\
\hline Negative & 239 & $98(17.56)$ & $141(28.43)$ & & \\
\hline Positive & 813 & $459(82.26)$ & 354 (71.37) & & \\
\hline PR status & & & & 30.3951 & $0.0005^{\mathrm{a}}$ \\
\hline Indeterminate & 4 & $2(0.36)$ & $2(0.4)$ & & \\
\hline Negative & 345 & $141(25.27)$ & $204(41.21)$ & & \\
\hline Positive & 704 & $415(74.37)$ & $289(58.38)$ & & \\
\hline HER2 status & & & & 10.4688 & $0.0185^{\mathrm{b}}$ \\
\hline Equivocal & 180 & $90(18.95)$ & $90(20.18)$ & & \\
\hline Indeterminate & 12 & $9(1.89)$ & $3(0.67)$ & & \\
\hline Negative & 565 & $307(64.63)$ & $258(57.85)$ & & \\
\hline Positive & 164 & $69(14.53)$ & $95(21.3)$ & & \\
\hline Menopause status & & & & 5.2218 & 0.1549 \\
\hline Indeterminate & 34 & $14(2.61)$ & $20(4.22)$ & & \\
\hline Peri & 40 & $21(3.91)$ & $19(4.01)$ & & \\
\hline Post & 706 & $390(72.63)$ & $316(66.67)$ & & \\
\hline Pre & 231 & $112(20.86)$ & $119(25.11)$ & & \\
\hline $\mathrm{T}$ classification & & & & 7.3029 & 0.1014 \\
\hline $\mathrm{T} 1$ & 281 & $163(27.82)$ & $118(22.87)$ & & \\
\hline $\mathrm{T} 2$ & 640 & $321(54.78)$ & $319(61.82)$ & & \\
\hline $\mathrm{T} 3$ & 138 & $81(13.82)$ & $57(11.05)$ & & \\
\hline $\mathrm{T} 4$ & 40 & $20(3.41)$ & $20(3.88)$ & & \\
\hline $\mathrm{TX}$ & 3 & $1(0.17)$ & $2(0.39)$ & & \\
\hline $\mathrm{N}$ classification & & & & 8.1021 & 0.0985 \\
\hline N0 & 516 & $284(48.46)$ & $232(44.96)$ & & \\
\hline $\mathrm{N} 1$ & 367 & $196(33.45)$ & $171(33.14)$ & & \\
\hline $\mathrm{N} 2$ & 120 & $53(9.04)$ & 67 (12.98) & & \\
\hline N3 & 79 & $46(7.85)$ & $33(6.4)$ & & \\
\hline NX & 20 & $7(1.19)$ & $13(2.52)$ & & \\
\hline M classification & & & & 8.9899 & $0.0090^{\mathrm{a}}$ \\
\hline M0 & 917 & $472(80.55)$ & 445 (86.24) & & \\
\hline M1 & 22 & $10(1.71)$ & $12(2.33)$ & & \\
\hline MX & 163 & $104(17.75)$ & $59(11.43)$ & & \\
\hline
\end{tabular}


Table II. Continued.

UBA7 mRNA expression

\begin{tabular}{|c|c|c|c|c|c|}
\hline & & & & & \\
\hline Characteristics & Patients, $\mathrm{n}$ & High, n (\%) & Low, n (\%) & $\chi^{2}$ & P-value \\
\hline Stage & & & & 6.3959 & 0.1714 \\
\hline I & 182 & $106(18.24)$ & $76(14.81)$ & & \\
\hline II & 626 & $334(57.49)$ & $292(56.92)$ & & \\
\hline III & 252 & $128(22.03)$ & $124(24.17)$ & & \\
\hline IV & 20 & $9(1.55)$ & $11(2.14)$ & & \\
\hline $\mathrm{X}$ & 14 & $4(0.69)$ & $10(1.95)$ & & \\
\hline Lymph node status & & & & 0.0215 & 1.0000 \\
\hline No & 28 & $16(3.77)$ & $12(3.99)$ & & \\
\hline Yes & 697 & $408(96.23)$ & $289(96.01)$ & & \\
\hline Margin status & & & & 0.4795 & 0.7816 \\
\hline Close & 31 & $15(2.7)$ & $16(3.35)$ & & \\
\hline Negative & 922 & $496(89.37)$ & $426(89.31)$ & & \\
\hline Positive & 79 & $44(7.93)$ & $35(7.34)$ & & \\
\hline Vital status & & & & 19.4879 & $0.0005^{\mathrm{a}}$ \\
\hline Deceased & 155 & $57(9.73)$ & $98(18.99)$ & & \\
\hline Living & 947 & $529(90.27)$ & $418(81.01)$ & & \\
\hline Radiation therapy & & & & 0.0058 & 0.9535 \\
\hline No & 445 & $241(44.3)$ & $204(44.54)$ & & \\
\hline Yes & 557 & $303(55.7)$ & $254(55.46)$ & & \\
\hline Neoadjuvant treatment & & & & 2.9898 & 0.0925 \\
\hline No & 1088 & $575(98.29)$ & $513(99.42)$ & & \\
\hline Yes & 13 & $10(1.71)$ & $3(0.58)$ & & \\
\hline Targeted therapy & & & & 0.1257 & 0.7571 \\
\hline No & 46 & $23(8.36)$ & $23(7.57)$ & & \\
\hline Yes & 533 & $252(91.64)$ & $281(92.43)$ & & \\
\hline Sample type & & & & 0.9341 & 0.4663 \\
\hline Metastatic & 7 & $5(0.85)$ & $2(0.39)$ & & \\
\hline Primary tumor & 1097 & $583(99.15)$ & $514(99.61)$ & & \\
\hline Overall survival & & & & 19.9101 & $0.0005^{\mathrm{a}}$ \\
\hline No & 933 & $520(90.28)$ & $413(80.82)$ & & \\
\hline Yes & 154 & $56(9.72)$ & 98 (19.18) & & \\
\hline Relapse-free survival & & & & 10.6443 & $0.0005^{\mathrm{a}}$ \\
\hline No & 816 & $474(92.4)$ & $342(85.71)$ & & \\
\hline Yes & 96 & $39(7.6)$ & $57(14.29)$ & & \\
\hline
\end{tabular}

${ }^{\mathrm{a}} \mathrm{P}<0.01 ;{ }^{\mathrm{b}} \mathrm{P}<0.05$. ER, estrogen receptor; $\mathrm{PR}$, progesterone receptor; HER2, human epidermal growth factor receptor 2; UBA7, ubiquitin-like modifier-activating enzyme 7 .

$M$ classification $(\mathrm{P}=0.0090)$, vital status $(\mathrm{P}=0.0005)$, OS $(\mathrm{P}=0.0005)$ and RFS $(\mathrm{P}=0.0005$; Table II).

Diagnostic capability of UBA7. ROC curves were generated to determine the diagnostic ability of UBA7 in breast cancer (Fig.3). The area under the curve (AUC) value was 0.559 (Fig. 3A), which indicates a reasonable diagnostic ability. Subsequently, subgroup analysis of different stages was performed and all data presented an adequate diagnostic ability ( 0.540 for stage I, 0.562 for stage II, 0.557 for stage III and 0.551 for stage IV; Fig. 3B-E).
Low UBA7 expression is associated with poor survival. Kaplan-Meier curves were generated and a log-rank test was performed to evaluate the prognostic value of UBA7 in breast cancer, and OS and RFS were used as prognostic parameters. The results demonstrated that patients with low UBA7 expression levels had poorer OS ( $\mathrm{P}<0.0001$; Fig. 4A). Further molecular subtype analysis demonstrated that patients with luminal A breast cancer $(\mathrm{P}=0.0210$; Fig. 4B), luminal $\mathrm{B}$ breast cancer $(\mathrm{P}=0.0054$; Fig. $4 \mathrm{C})$, infiltrating ductal carcinoma $\left(\mathrm{P}=1.4 \times 10^{-4}\right.$; Fig. $\left.4 \mathrm{~F}\right)$ or infiltrating lobular carcinoma 
Table III. Univariate and multivariate analyses of overall survival in patients with breast cancer.

\begin{tabular}{|c|c|c|c|c|c|c|}
\hline \multirow[b]{2}{*}{ Characteristics } & \multicolumn{3}{|c|}{ Univariate analysis } & \multicolumn{3}{|c|}{ Multivariate analysis } \\
\hline & HR & $95 \% \mathrm{CI}$ & P-value & HR & $95 \% \mathrm{CI}$ & P-value \\
\hline Age, years & 1.91 & $1.39-2.63$ & $<0.001^{\mathrm{a}}$ & 2.31 & $1.44-3.68$ & $<0.001^{\mathrm{a}}$ \\
\hline Histological type & 0.93 & $0.74-1.17$ & 0.543 & & & \\
\hline Molecular subtype & 1.01 & $0.88-1.16$ & 0.901 & & & \\
\hline ER & 0.85 & $0.71-1.02$ & 0.074 & & & \\
\hline PR & 0.87 & $0.73-1.03$ & 0.096 & & & \\
\hline HER2 & 1.29 & $1.05-1.57$ & $0.013^{\mathrm{b}}$ & 1.10 & $0.88-1.37$ & 0.3840 \\
\hline Menopause status & 1.16 & $0.94-1.43$ & 0.165 & & & \\
\hline Stage & 1.64 & $1.40-1.91$ & $<0.001^{\mathrm{a}}$ & 2.12 & $1.60-2.80$ & $<0.001^{\mathrm{a}}$ \\
\hline Lymph node status & 1.10 & $0.93-1.30$ & 0.274 & & & \\
\hline Margin status & 1.42 & $1.11-1.81$ & $0.005^{\mathrm{a}}$ & 0.98 & $0.70-1.38$ & 0.9290 \\
\hline UBA7 & 2.09 & $1.50-2.90$ & $<0.001^{\mathrm{a}}$ & 2.10 & $1.30-3.39$ & $0.0020^{\mathrm{a}}$ \\
\hline
\end{tabular}

${ }^{\mathrm{a}} \mathrm{P}<0.01$; ${ }^{\mathrm{P}}<0.05$. HR, hazard ratio; CI, confidence interval; ER, estrogen receptor; PR, progesterone receptor; HER2, human epidermal growth factor receptor 2; UBA7, ubiquitin-like modifier-activating enzyme 7.
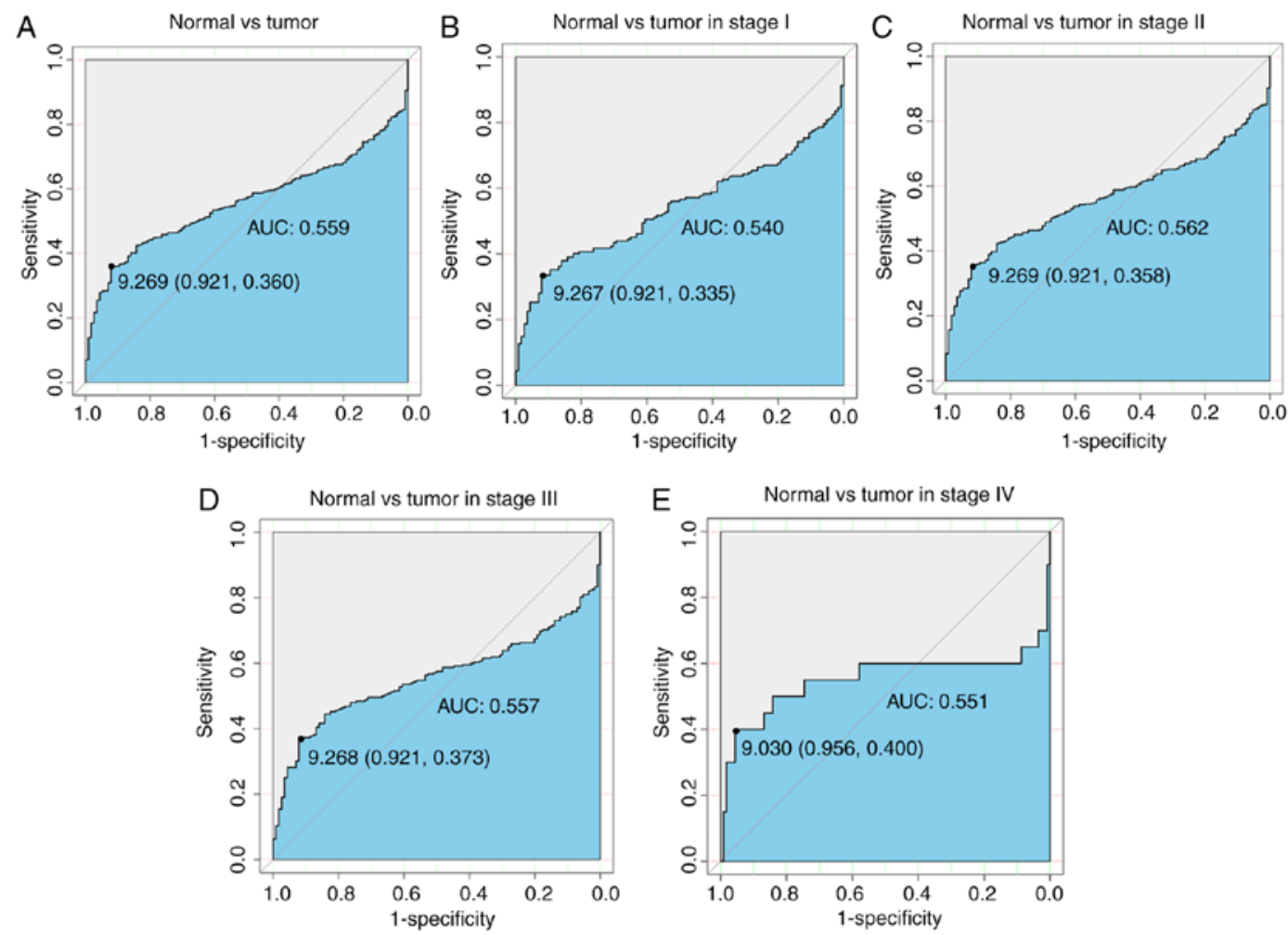

Figure 3. Receiver operating characteristic curves of UBA7 in different clinical stages. UBA7 exhibited mild diagnostic ability. (A) The AUC is 0.559 , which represents a reasonable diagnostic ability. (B-E) Subgroup analysis of different stages also demonstrated a reasonable diagnostic ability. UBA7, ubiquitin-like modifier-activating enzyme 7; AUC, area under the curve.

$(\mathrm{P}=0.0033$; Fig. $4 \mathrm{G})$ had poorer $\mathrm{OS}$ in the presence of low UBA7 expression. Univariate Cox analysis identified the critical variables associated with OS, including age $(\mathrm{P}<0.001)$, HER2 expression $(\mathrm{P}=0.0130)$, stage $(\mathrm{P}<0.001)$, margin status $(\mathrm{P}=0.0050)$ and UBA7 expression $(\mathrm{P}<0.001)$ (Table III). Multivariate Cox analysis indicated that age [hazard ratio (HR), 2.31; $\mathrm{P}<0.001)$, stage (HR, 2.12; $\mathrm{P}<0.001)$ and UBA7 expression (HR, 2.10; $\mathrm{P}=0.0020)$ may serve as independent prognostic factors, as presented in Table III.

Patients with low UBA7 expression levels also had a poor prognosis in terms of RFS ( $\mathrm{P}=0.0011$; Fig. 5A). Furthermore, low UBA7 expression was associated with poor RFS in patients with luminal $\mathrm{B}$ breast cancer $(\mathrm{P}<0.0001$; Fig. 5C), infiltrating ductal carcinoma ( $\mathrm{P}=0.0031$; Fig. 5F) 
Table IV. Univariate and multivariate analyses of relapse-free survival in patients with breast cancer.

\begin{tabular}{|c|c|c|c|c|c|c|}
\hline \multirow[b]{2}{*}{ Characteristics } & \multicolumn{3}{|c|}{ Univariate analysis } & \multicolumn{3}{|c|}{ Multivariate analysis } \\
\hline & $\mathrm{HR}$ & $95 \% \mathrm{CI}$ & P-value & HR & $95 \% \mathrm{CI}$ & $\mathrm{P}$-value \\
\hline Age, years & 1.45 & $0.97-2.16$ & 0.072 & & & \\
\hline Histological type & 0.86 & $0.65-1.14$ & 0.290 & & & \\
\hline Molecular subtype & 0.99 & $0.82-1.20$ & 0.945 & & & \\
\hline ER & 0.78 & $0.63-0.97$ & $0.026^{\mathrm{a}}$ & 0.88 & $0.63-1.22$ & 0.441 \\
\hline PR & 0.78 & $0.64-0.96$ & $0.019^{\mathrm{a}}$ & 0.87 & $0.64-1.18$ & 0.360 \\
\hline HER2 & 0.93 & $0.70-1.22$ & 0.596 & & & \\
\hline Menopause status & 0.95 & $0.74-1.22$ & 0.713 & & & \\
\hline Stage & 1.71 & $1.40-2.08$ & $<0.001^{\mathrm{b}}$ & 1.55 & $1.24-1.92$ & $<0.001^{\mathrm{b}}$ \\
\hline Lymph node status & 0.86 & $0.70-1.06$ & 0.159 & & & \\
\hline Margin status & 1.59 & $1.23-2.06$ & $<0.001^{\mathrm{b}}$ & 1.48 & $1.13-1.94$ & $0.005^{\mathrm{b}}$ \\
\hline UBA7 & 1.95 & $1.30-2.93$ & $0.001^{\mathrm{b}}$ & 1.59 & $1.03-2.45$ & $0.037^{\mathrm{a}}$ \\
\hline
\end{tabular}

${ }^{\mathrm{a}} \mathrm{P}<0.05 ;{ }^{\mathrm{b}} \mathrm{P}<0.01$. HR, hazard ratio; $\mathrm{CI}$, confidence interval; ER, estrogen receptor; PR, progesterone receptor; HER2, human epidermal growth factor receptor 2; UBA7, ubiquitin-like modifier-activating enzyme.

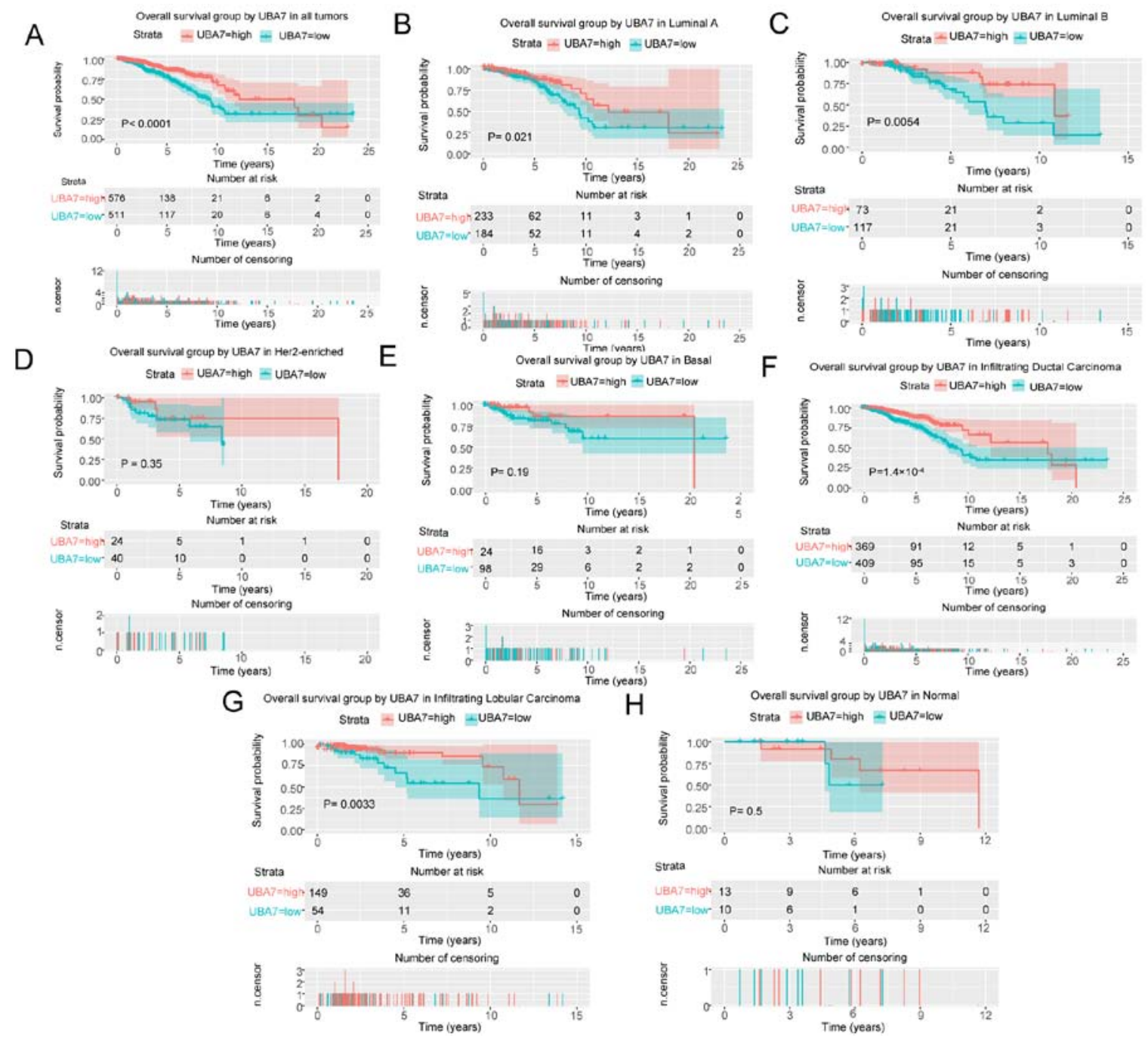

Figure 4. Analysis of the association between UBA7 expression and OS. (A) Patients with low UBA7 expression had poor OS. Patients with (B) Luminal A breast cancer, (C) Luminal B breast cancer, $(F)$ infiltrating ductal carcinoma and $(G)$ infiltrating lobular carcinoma had poor OS in the presence of low UBA7 expression. However, no significant associations were observed between UBA7 expression and OS in patients with (D) HER2-enriched breast cancer and (E) basal-like breast cancer, and in (H) normal cases. UBA7, ubiquitin-like modifier-activating enzyme 7; OS, overall survival; HER2, human epidermal growth factor receptor 2. 

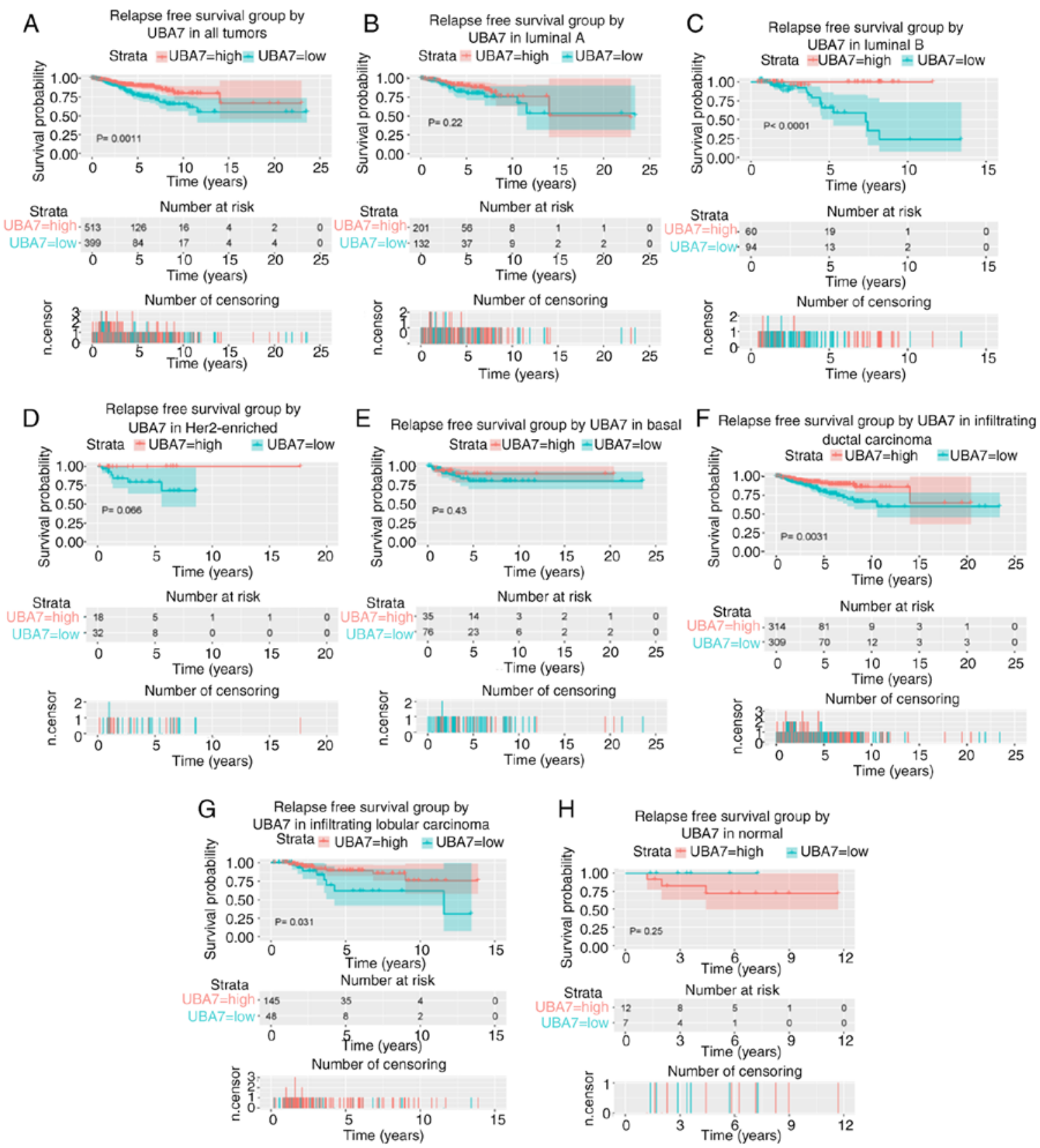

Figure 5. Survival analysis of UBA7 expression in terms of RFS. (A) Patients with low UBA7 expression had a poor prognosis in terms of RFS. Low UBA7 expression was significantly associated with poor RFS in patients with (C) Luminal B breast cancer, (F) infiltrating ductal carcinoma and (G) infiltrating lobular carcinoma. However, no significant associations were observed between UBA7 expression and RFS in patients with (B) Luminal A breast cancer, (D) HER2-enriched breast cancer and (E) basal-like breast cancer, and in (H) normal cases. UBA7, ubiquitin-like modifier-activating enzyme 7; RFS, relapse-free survival; HER2, human epidermal growth factor receptor 2.

and infiltrating lobular carcinoma ( $\mathrm{P}=0.0310$; Fig. 5G). Univariate Cox analysis and multivariate Cox analysis indicated that stage $(\mathrm{P}<0.001)$, margin status $(\mathrm{P}=0.0050)$ and UBA7 expression $(\mathrm{P}=0.0370)$ were independent prognostic factors for poor RFS (Table IV).

Kaplan-Meier curves were generated using the log-rank test to further investigate the association between UBA7 expression and the expression levels of ER, PR and HER2 in terms of OS (Fig. 6) and RFS (Fig. 7). The results demonstrated patients with ER-positive $\left(\mathrm{P}=1.8 \times 10^{-4}\right.$; Fig. $\left.6 \mathrm{~B}\right)$. PR-negative $(\mathrm{P}=0.0180$; Fig. 6C), PR-positive $(\mathrm{P}=0.0031$; Fig. 6D) and HER2-negative $(\mathrm{P}=0.0180$; Fig. $6 \mathrm{E})$ breast cancer had poor OS in the presence of low UBA7 expression. Furthermore, patients with ER-positive ( $\mathrm{P}=0.0066$; Fig. 7B) and PR-negative $(\mathrm{P}=0.0350$; Fig. 7C) breast cancer also had poor RFS in the presence of low UBA7 expression.

\section{Discussion}

Previous studies have set out to identify novel biomarkers for the diagnosis and prognosis of different diseases (15-24); however, to the best of our knowledge, the present study was the first to demonstrate that UBA7 may function as a biomarker in breast cancer. The results demonstrated that UBA7 expression was decreased in breast cancer compared with that in normal tissues. Furthermore, UBA7 expression differences were 
A
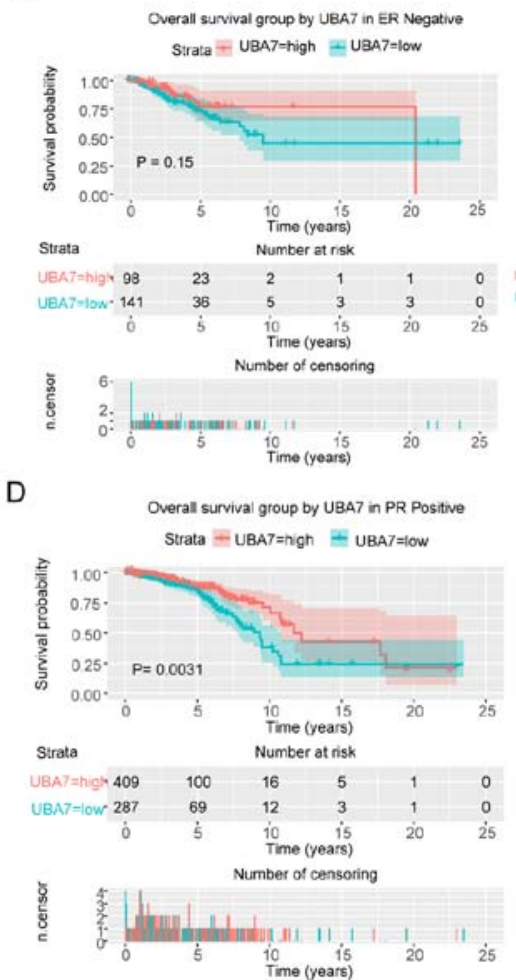

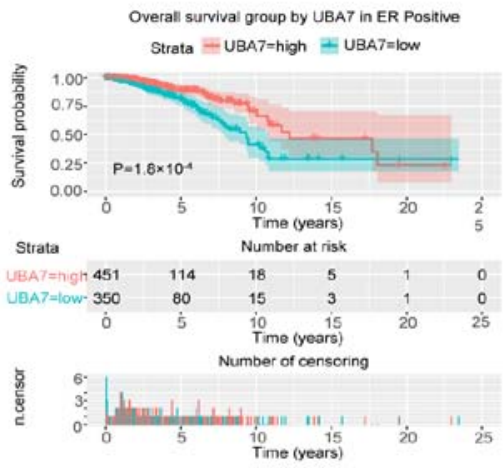

E

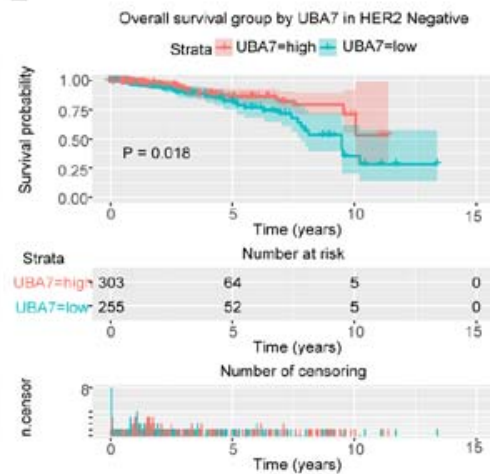

C

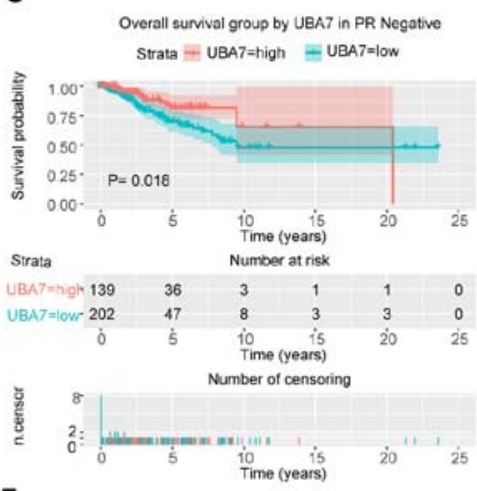

$\mathrm{F}$

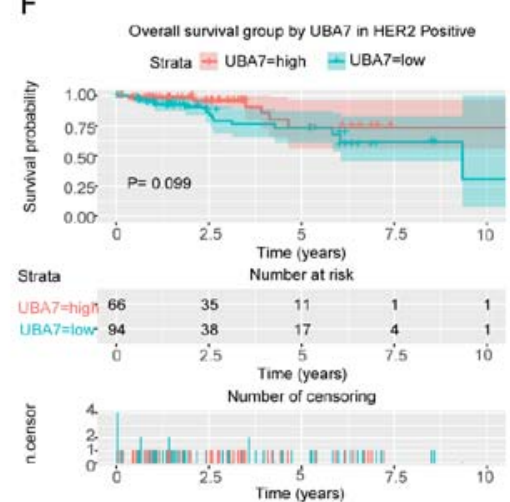

Figure 6. Survival analysis of UBA7 expression in terms of OS, according to the expression of ER, PR and HER2. Low UBA7 expression was significantly associated with poor OS in (B) ER-positive, PR (C) -negative and (D) -positive, and (E) HER2-negative patients. However, no significant associations were observed between low UBA7 expression and OS in (A) ER-negative and (F) HER2-positive patients. UBA7, ubiquitin-like modifier-activating enzyme 7; OS, overall survival; ER, estrogen receptor; PR, progesterone receptor; HER2, human epidermal growth factor receptor 2.

A
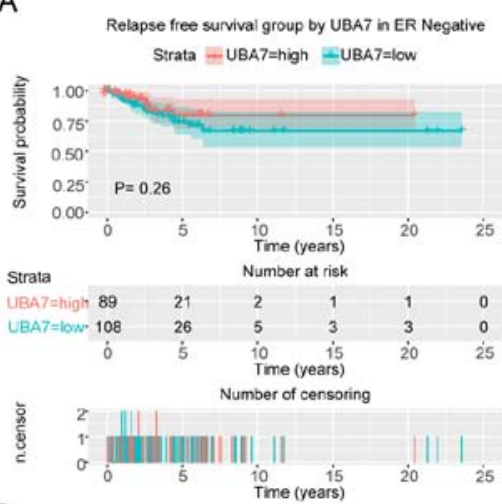

D

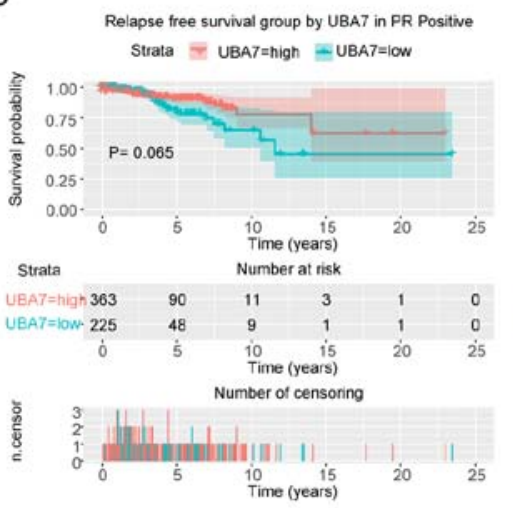

B
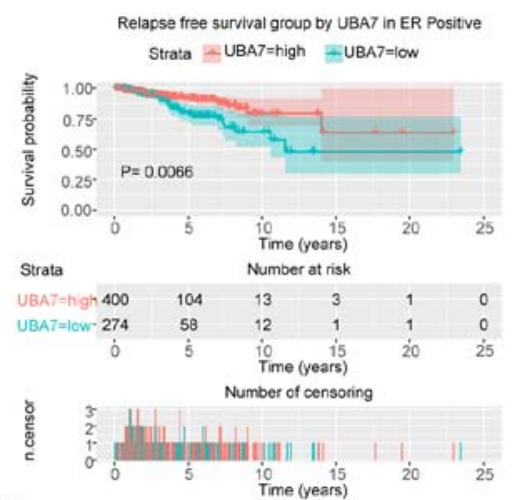

$\mathrm{E}$

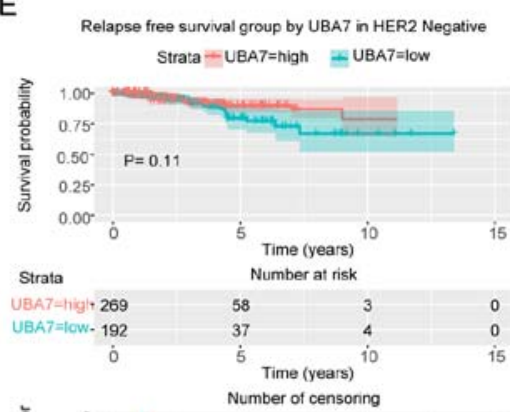

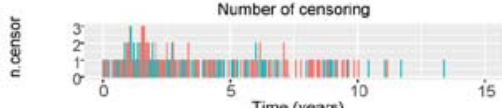

C

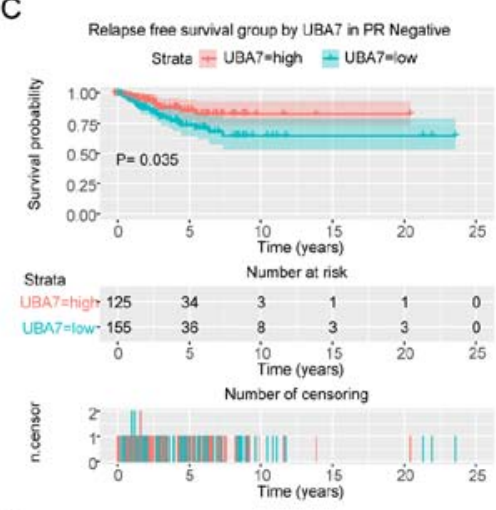

$\mathrm{F}$

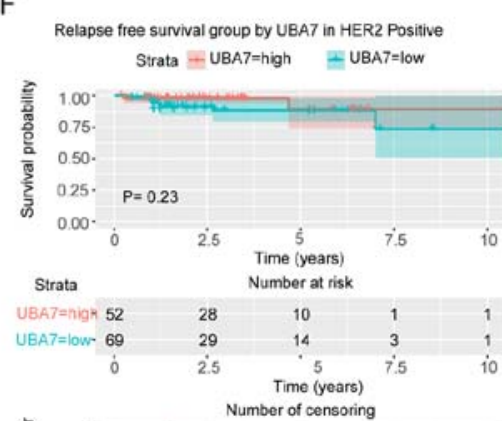

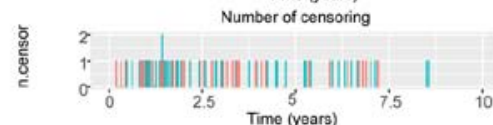

Figure 7. Survival analysis of UBA7 expression in terms of RFS, according to the expression of ER, PR and HER2. Low UBA7 expression was significantly associated with poor RFS in (B) ER-positive and (C) PR-negative patients. However, no significant associations were observed between low UBA7 expression and RFS in (A) ER-negative, (D) PR-positive, and HER2 (E) -negative and (F) -positive patients. UBA7, ubiquitin-like modifier-activating enzyme 7; RFS, relapse-free survival; ER, estrogen receptor; PR, progesterone receptor; HER2, human epidermal growth factor receptor 2. 
associated with vital status, tumor classification, metastasis classification, histological type, sex, molecular subtype, and the expression of PR, ER and HER2. The low and high UBA7 expression groups also demonstrated differences in some clinical characteristics, including molecular subtype, histological subtype and expression of ER, PR and HER2, vital status, OS and RFS. Patients with lower UBA7 expression had a poor prognosis. Although UBA7 demonstrated a reasonable diagnostic capability, it may still be used in combination with other markers to diagnose breast cancer.

Currently, the only known biological function of UBA7 is catalysing ISG15 conjugation. Previous studies have reported that UBA7 has the potential to act as a tumor suppressor gene in human lung cancer (8-12). UBA7 is located on human chromosome region $3 \mathrm{p} 21.3$, and $\mathrm{LOH}$ on chromosome $3 \mathrm{p} 21.3$ has been observed in 70-80\% of NSCLC and $90-100 \%$ of SCLC cases (8). The expression levels of UBA7, previously referred to as UBE1L, have been reported to be markedly decreased in several lung cancer cell lines $(11,25,26)$. Notably, Kok et al (27) demonstrated that although one allele of UBA7 was absent in lung cancer cell lines, the remaining allele was still detectable and without mutation (27). The results of the present study demonstrated that UBA7 expression was significantly decreased in breast cancer and that stage IV tumors had the lowest UBA7 expression levels. Furthermore, UBA7 expression was also associated with clinical characteristics, and low UBA7 expression was demonstrated to be associated with poor prognosis, especially in patients with ER-positive and PR-positive breast cancer.

A number of studies have demonstrated that UBA7 can promote the formation of a complex between ISG15 and cyclin D1, and inhibit cyclin D1, which is associated with cancer cell proliferation (8-12). UBA7-knockdown has been reported to increase cyclin D1 expression; however, overexpression of UBA7 has been shown to reduce cyclin D1 expression and suppress lung cancer cell proliferation (9). Furthermore, it has been reported that UBA7 can suppress EGFR at the post-translational level in HBE-Beas-2B cells and suppress the subsequent $\mathrm{AKT} / \mathrm{NF}-\kappa \mathrm{B}$ signaling pathway (12). However, deficiency of UBA7 has no effect in K-ras (LA2)-mutant mice with lung cancer, thus UBA7 is not considered a tumor suppressor gene in the K-ras lung cancer mouse model (8). In the present study, the decreased UBA7 expression levels in breast cancer were not as obvious as it was demonstrated to be in lung cancer, thus it is suggested that UBA7 may not have the same effect on breast cancer cells. Furthermore, the results of the present study demonstrated that UBA7 was associated with the expression of PR, ER and HER2, and the molecular subtypes of breast cancer, thus UBA7 may have the potential to influence the proliferation of breast cancer cells through these molecules, which are closely associated with breast cancer. The association between UBA7 expression and cyclin D1, EGFR, ER, PR, HER2 and other tumor-associated molecules requires further experimental evidence; however, UBA7 may function as a target in treatment according to current data on lung cancer.

The molecular mechanism underlying UBA7 regulation remains unclear; however, a number of molecules have been reported to influence UBA7 expression. For example, IFN- $\alpha$ has the ability to induce ISGylation-associated protein expression, including UBA7 expression (28). Furthermore, retinoic acid has been demonstrated to induce UBA7 expression, which in turn induces ISGylation of promyelocytic leukaemia/retinoic acid receptor- $\alpha$ protein and causes degradation in acute promyelocytic leukaemia (29). Previous studies have reported that chemopreventive polyphenols $(10,30,31)$, such as curcumin, epigallocatechin gallate and resveratrol, have the ability to influence UBA7 expression by altering the intracellular reactive oxygen species status, which is regulated via the c-Jun N-terminal kinase/nuclear factor erythroid 2-related factor 2 signaling pathway; however, this association exhibits dose-dependent effects (10). IFNs have been used with the intent to cure cancer for several years, including breast cancer (30). IFNs have been reported to exert antitumor effects by regulating the immune system and affecting cell proliferation and apoptosis (31). A limitation of the present study was failure to assess the influence of IFNs on UBA7 expression. Thus, future studies will focus on the use of IFNs and other molecules, including chemopreventive polyphenols, which may influence UBA7 expression, while studying the change in UBA7 expression in breast cancer, and will aim to investigate whether IFNs suppress breast cancer through the UBA7 pathway.

Although UBA7 has been considered a potential tumor suppressor gene in lung cancer for decades, its underlying molecular mechanism still remains unclear. Furthermore, the role of UBA7 in different types of cancer still lacks sufficient evidence to support this hypothesis. To the best of our knowledge, the present study was the first to uncover UBA7 expression changes in breast cancer. The results demonstrated that UBA7 expression was decreased in breast cancer and was also associated with clinical characteristics. In addition, low UBA7 expression was associated with a poor prognosis. Taken together, these results suggest that UBA7 may function as a biomarker for the clinical diagnosis and prognosis of breast cancer, and even as a target in treatment.

The present study aimed to identify the changes in UBA7 expression in breast cancer using bioinformatic methods from a macro perspective. Further studies and evidence from the laboratory are required to support the stated speculations, and the potential molecular mechanisms also need to be investigated. The subsequent stages of research have already been designed, and will focus on laboratory work, including the collection of tissues from patients, analysis of UBA7 and other related molecules expression levels in tissues, and the exploration of associations between molecules and clinical features, and even potential molecular mechanisms using cells.

\section{Acknowledgements}

Not applicable.

\section{Funding}

No funding was received.

\section{Availability of data and material}

The datasets generated and analyzed during the present study were obtained from The Cancer Genome Atlas (TCGA) database (https://cancergenome.nih.gov/). 


\section{Authors' contributions}

ML and YL designed the study and wrote the manuscript. YJ and FH had a significant role in the study design, data collection and manuscript review. SQ and YJ acquired and analysed the data. All authors read and approved the final manuscript.

\section{Ethics approval and consent to participate}

Not applicable.

\section{Patient consent for publication}

Not applicable.

\section{Competing interests}

The authors declare that they have no competing interests.

\section{References}

1. Howlader N, Noone AM, Krapcho M, Miller D, Brest A, Yu M, Ruhl J, Tatalovich Z, Mariotto A, Lewis DR, Chen HS, Feuer EJ and Cronin KA (eds). Lifetime risk (Percent) of dying from cancer by site and race/ethnicity: Female, Total U.S., 2014-2016. In: SEER Cancer Statistics Review, 1975-2016. National Cancer Institute, Bethesda, MD, USA.

2. American Cancer Society: Breast Cancer Facts \& Figures 2017-2018. American Cancer Society, Inc., Atlanta, GA, 2017.

3. Zhang X, Bogunovic D, Payelle-Brogard B, Francois-Newton V, Speer SD, Yuan C, Volpi S, Li Z, Sanal O, Mansouri D, et al: Human intracellular ISG15 prevents interferon- $\alpha / \beta$ over-amplification and auto-inflammation. Nature 517: 89-93, 2015.

4. Jeon YJ, Yoo HM and Chung CH: ISG15 and immune diseases. Biochim Biophys Acta 1802: 485-496, 2010.

5. Zhou MJ, Chen FZ, Chen HC, Wan XX, Zhou X, Fang Q and Zhang DZ: ISG15 inhibits cancer cell growth and promotes apoptosis. Int J Mol Med 39: 446-452, 2017.

6. Zhao C, Denison C, Huibregtse JM, Gygi S and Krug RM: Human ISG15 conjugation targets both IFN-induced and constitutively expressed proteins functioning in diverse cellular pathways. Proc Natl Acad Sci USA 102: 10200-10205, 2005.

7. Darb-Esfahani S, Sinn BV, Rudl M, Sehouli J, Braicu I, Dietel M and Denkert C: Interferon-stimulated gene, $15 \mathrm{kDa}$ (ISG15) in ovarian high-grade serous carcinoma: Prognostic impact and link to NF-кB pathway. Int J Gynecol Pathol 33: 16-22, 2014.

8. Yin X, Cong X, Yan M and Zhang DE: Deficiency of a potential 3p21.3 tumor suppressor gene UBE1L (UBA7) does not accelerate lung cancer development in K-rasLA2 mice. Lung Cancer 63: 194-200, 2009.

9. Feng Q, Sekula D, Guo Y, Liu X, Black CC, Galimberti F, Shah SJ, Sempere LF, Memoli V, Andersen JB, et al: UBE1L causes lung cancer growth suppression by targeting cyclin D1. Mol Cancer Ther 7: 3780-3788, 2008.

10. Jiang A, Li Y, Wang P, Shan X, Jiang P, Wang X and Feng Q: Mechanism of dose-dependent regulation of UBE1L by polyphenols in human bronchial epithelial cells. J Cell Biochem 116: $1553-1562,2015$

11. Pitha-Rowe I, Petty WJ, Feng Q, Koza-Taylor PH, Dimattia DA, Pinder L, Dragnev KH, Memoli N, Memoli V, Turi T, et al: Microarray analyses uncover UBE1L as a candidate target gene for lung cancer chemoprevention. Cancer Res 64: 8109-8115, 2004

12. Jiang AP, Zhou DH, Meng XL, Zhang AP, Zhang C, Li XT and Feng Q: Down-regulation of epidermal growth factor receptor by curcumin-induced UBE1L in human bronchial epithelial cells. J Nutr Biochem 25: 241-249, 2014
13. Samur MK: RTCGAToolbox: A new tool for exporting TCGA Firehose data. PLoS One 9: e106397, 2014.

14. R Core Team: A language and environment for statistical computing. R Foundation for Statistical Computing, Vienna, Austria, 2012. Available online at https://www.R-project.org/.

15. Jiao Y, Fu Z, Li Y, Meng L and Liu Y: High EIF2B5 mRNA expression and its prognostic significance in liver cancer: A study based on the TCGA and GEO database. Cancer Manag Res 10: 6003-6014, 2018.

16. Jiao Y, Li Y, Lu Z and Liu Y: High trophinin-associated protein expression is an independent predictor of poor survival in liver cancer. Dig Dis Sci 64: 137-143, 2019.

17. Jiao Y, Fu Z, Li Y, Zhang W and Liu Y: Aberrant FAM64A mRNA expression is an independent predictor of poor survival in pancreatic cancer. PLoS One 14: e0211291, 2019.

18. Jiao Y, Li Y, Jiang P, Han W and Liu Y: PGM5: A novel diagnostic and prognostic biomarker for liver cancer. PeerJ 7: e7070, 2019.

19. Jiao Y, Li Y, Liu S, Chen Q and Liu Y: ITGA3 serves as a diagnostic and prognostic biomarker for pancreatic cancer. Onco Targets Ther 12: 4141-4152, 2019.

20. Hou L, Zhang X, Jiao Y, Li Y, Zhao Y, Guan Y and Liu Z: ATP binding cassette subfamily B member 9 (ABCB9) is a prognostic indicator of overall survival in ovarian cancer. Medicine (Baltimore) 98: e15698, 2019.

21. Sun Z, Sun L, He M, Pang Y, Yang Z and Wang J: Low BCL7A expression predicts poor prognosis in ovarian cancer. J Ovarian Res 12: 41, 2019.

22. Li Y, Jiao Y, Fu Z, Luo Z, Su J and Li Y: High miR-454-3p expression predicts poor prognosis in hepatocellular carcinoma. Cancer Manag Res 11: 2795-2802, 2019.

23. Cai H, Jiao Y, Li Y, Yang Z, He M and Liu Y: Low CYP24A1 mRNA expression and its role in prognosis of breast cancer. Sci Rep 9: 13714, 2019.

24. Cui Y, Jiao Y, Wang K, He M and Yang Z: A new prognostic factor of breast cancer: High carboxyl ester lipase expression related to poor survival. Cancer Genet 239: 54-61, 2019.

25. Kok K, Hofstra R, Pilz A, van den Berg A, Terpstra P, Buys CH and Carritt B: A gene in the chromosomal region 3p21 with greatly reduced expression in lung cancer is similar to the gene for ubiquitin-activating enzyme. Proc Natl Acad Sci USA 90: 6071-6075, 1993

26. McLaughlin PM, Helfrich W, Kok K, Mulder M, Hu SW, Brinker MG, Ruiters MH, de Leij LF and Buys CH: The ubiquitin-activating enzyme El-like protein in lung cancer cell lines. Int J Cancer 85: 871-876, 2000.

27. Kok K, Van den Berg A, Veldhuis PM, Franke M, Terpstra P and Buys CH: The genomic structure of the human UBE1L gene. Gene Expr 4: 163-175, 1995.

28. Borsini A, Cattaneo A, Malpighi C, Thuret S, Harrison NA; MRC ImmunoPsychiatry Consortium, Zunszain PA and Pariante CM: Interferon-alpha reduces human hippocampal neurogenesis and increases apoptosis via activation of distinct STAT1-dependent mechanisms. Int J Neuropsychopharmacol 21: 187-200, 2018.

29. Kitareewan S, Pitha-Rowe I, Sekula D, Lowrey CH, Nemeth MJ, Golub TR, Freemantle SJ and Dmitrovsky E: UBE1L is a retinoid target that triggers PML/RARalpha degradation and apoptosis in acute promyelocytic leukemia. Proc Natl Acad Sci USA 99: 3806-3811, 2002.

30. Carpi A, Nicolini A, Antonelli A, Ferrari P and Rossi G: Cytokines in the management of high risk or advanced breast cancer: An update and expectation. Curr Cancer Drug Targets 9: 888-903, 2009.

31. Ren WB, Xia XJ, Huang J, Guo WF, Che YY, Huang TH and Lei LC: Interferon- $\gamma$ regulates cell malignant growth via the c-Abl/HDAC2 signaling pathway in mammary epithelial cells. J Zhejiang Univ Sci B 20: 39-48, 2019.

This work is licensed under a Creative Commons Attribution-NonCommercial-NoDerivatives 4.0 International (CC BY-NC-ND 4.0) License. 